\title{
Micromagnetics of nanostructures
}

\author{
N.A. Usova,*, Yu.B. Grebenshchikov ${ }^{\mathrm{a}}$, L.G. Kurkina ${ }^{\mathrm{a}}$, Ching-Ray Chang ${ }^{\mathrm{b}}$, \\ Zung-Hang Wei ${ }^{\mathrm{b}}$
}

\author{
${ }^{a}$ Troitsk Institute for Innovation and Fusion Research, Troitsk, Moscow region 142190, Russia \\ b Department of Physics, National Taiwan University, Taipei, Taiwan 10617, Republic of China
}

\begin{abstract}
The results of numerical simulation of equilibrium magnetization patterns and magnetization reversal in flat cylindrical and elliptical particles of the soft magnetic type are discussed. It is found that the bending state originates in these particles due to instability of quasi-uniform magnetization at large enough in-plane sizes. Similar states exist also in certain intervals of an external magnetic field. It is shown that the magnetization reversal in an elliptical particle depends considerably on the particle thickness and aspect ratio. The nucleation field of an elliptical particle saturates as a function of particle aspect ratio. The effect of a mutual magnetostatic interaction on the magnetic properties of an array of soft magnetic particles is discussed briefly.
\end{abstract}

(C) 2002 Elsevier Science B.V. All rights reserved.

Keywords: Soft magnetic particles; Equilibrium magnetization patterns; Magnetization reversal

\section{Introduction}

Magnetic properties of thin ferromagnetic particles and patterned structures are very promising for various technological applications [1]. The results of recent experiments [2-6] clearly show that the behavior of magnetization vortices is responsible for static and dynamic properties of soft magnetic particles of submicron dimensions. Also, a difference is revealed in the magnetization reversal mechanisms for particles of rectangular and smooth elliptical shapes $[3,4,7]$. In contrast to the rectangular particles of submicron sizes where magnetization reversal always begins from the end domains [5,6], there is a possibility to create stable quasi-uniform state in an elliptical particle with large enough dimensions in the absence of an applied magnetic field. It was noted recently [8] that the investigation of stability of quasi-uniform magnetization as a function of particle size and material parameters is important in understanding magnetization reversal in elliptical or cylindrical particles. In the present paper, the results of numerical simulation of equilibrium

\footnotetext{
*Corresponding author. Fax: + 7-095-334-5776.

E-mail address: usov@obninsk.ru (N.A. Usov).
}

magnetization patterns and magnetization reversal in flat cylindrical and elliptical particles of soft magnetic type are discussed. In addition, the effect of mutual magnetostatic interaction on the magnetic properties of patterned structures is analyzed briefly.

\section{Equilibrium states}

For numerical simulations, the material parameters of a Permalloy particle (saturation magnetization $M_{\mathrm{s}}=$ $800 \mathrm{emu} / \mathrm{cm}^{3}$, exchange constant $C=2 A=2 \times 10^{-6} \mathrm{erg} /$ $\mathrm{cm}$, uniaxial anisotropy constant $K_{1}=10^{3} \mathrm{erg} / \mathrm{cm}^{3}$ ) are used. The particle is approximated by an array of small uniformly magnetized cubic cells with the size, $a=3-5 \mathrm{~nm}$, small enough with respect to the exchange length of the material, $R_{0}=C^{1 / 2} / M_{\mathrm{s}}=17.7 \mathrm{~nm}$. Equilibrium magnetization structures are obtained by means of a time integration of the Landau-Lifshitz-Gilbert equation according to the usual scheme $[9,10]$.

For the cylindrical particle, detailed numerical simulations are performed for thickness $L_{z}=12,18,24,30$ and $36 \mathrm{~nm}$ and for diameters $D \leqslant 210 \mathrm{~nm}$. As a typical example, the energy diagram of various stable 
magnetization states existing in a cylindrical particle with thickness $L_{z}=18 \mathrm{~nm}$ is shown in Fig. 1. For a small enough diameter, $D<D_{\text {sd }}$, a quasi-uniform (flower) state has the lowest total energy. Therefore, $D_{\text {sd }}$ can be considered as effective single-domain diameter of the cylindrical particle. One can see in Fig. 1 that the flower state remains stable up to another critical diameter, $D_{\mathrm{cl}}>D_{\mathrm{sd}}$. At $D>D_{\mathrm{cl}}$, a bending-type state develops gradually with increase in particle size. This state is denoted as BS 1 in Fig. 1. In contrast to the flower state, the magnetization of the bending state diminishes considerably as a function of the particle diameter. In turn, the BS 1 configuration becomes unstable at a critical diameter $D_{\mathrm{c} 2}$ due to the large curvature of the magnetization near the lateral side of the particle. This new instability leads to entering of the vortex inside the particle. As Fig. 1 shows, for thickness $L_{z}=18 \mathrm{~nm}$, another stable bending-type state, BS 2, with large in-plane magnetization is revealed numerically at larger diameters, $D \geqslant D_{\mathrm{c} 3}$. However, the BS 2 configuration is absent for thickness $L_{z} \geqslant 24 \mathrm{~nm}$. It is important to note also that the critical diameters $D_{\mathrm{c} 1}$ and $D_{\mathrm{c} 2}$ reduce gradually with increase in the particle thickness. Furthermore, there is no stable state with appreciable in-plane magnetization in cylindrical Permalloy particle with thickness $L_{z} \geqslant 36 \mathrm{~nm}$.

For the elliptical particle, the numerical simulations were carried out for fixed thickness $L_{z}=30 \mathrm{~nm}$, but for various aspect ratios, $1 \leqslant L_{x} / L_{y} \leqslant 4$. In addition to different vortex patterns studied earlier [8], stable bending states are obtained for the elliptical particle with sufficiently large in-plane dimensions. Similar to the case of cylindrical particle, the bending state in the elliptical particle arises due to instability of the quasiuniform magnetization at a certain critical transverse diameter. The latter depends on the particle aspect ratio. It is found that the average magnetization of the bending state decreases considerably as a function of the particle size at a fixed aspect ratio. Eventually, the

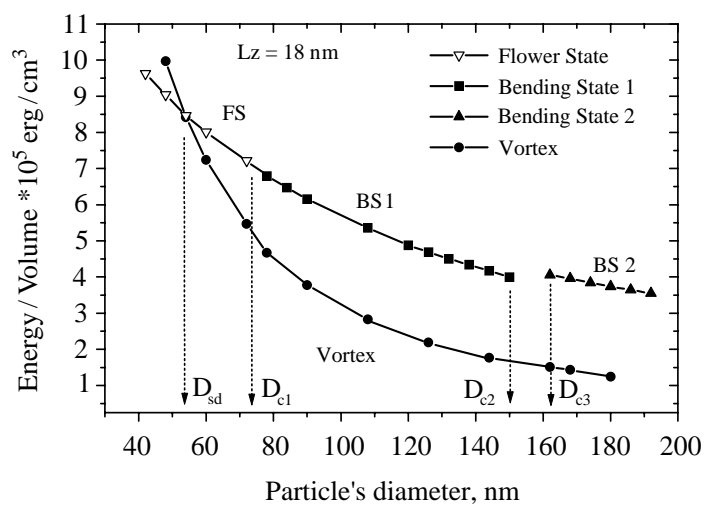

Fig. 1. Stable magnetization states in cylindrical Permalloy particle with thickness $L_{z}=18 \mathrm{~nm}$. bending state becomes unstable at another critical diameter where one or several vortices nucleate within the particle. The nucleation of the vortices occurs at the lateral side of the particle near the points where demagnetizing field reaches the value high enough to rotate the unit magnetization vector out of the particle plane. As the dynamical numerical simulation shows, the rotation of the magnetization occurs initially in a small volume with characteristic size of the order of exchange length. This process leads to the creation of a precursor of the vortex core at the lateral side of the particle. Later the vortex core moves gradually inside the particle forming a one-vortex or multi-vortex pattern. One can see, the bending states provide continuous transformation of the quasi-uniform magnetization into the vortex pattern with increase in the particle size. It should be noted, however, that the bending state is not a transient state. It can be stable in some intermediate range of sizes depending on the particle aspect ratio. It is found that for an elliptical particle with thickness $L_{z}=30 \mathrm{~nm}$, the ranges for stability of quasi-uniform and bending states increase rapidly as a function of the particle aspect ratio.

\section{Magnetization reversal}

We investigate also the magnetization reversal in elliptical particles with different thickness and aspect ratios. It is found that the magnetization reversal in the elliptical particle depends considerably on the particle thickness and aspect ratio. For an elongated elliptical particle, the magnetization reversal takes place usually in two different steps. Initially it starts due to instability of quasi-uniform magnetization in the reversed external magnetic field [8]. For an elliptical particle with aspect ratio $L_{x} / L_{y}>3$ and thickness $L_{z} / R_{0}>1$, the shape of the instability mode corresponds to that of a buckling mode in very long bar with the same width and thickness, [11]. However, the mode amplitude is suppressed considerably near the particle ends. Therefore, one may expect that for an elongated elliptical particle the edge roughness would have a minor effect on the magnetization reversal. At the second step of the magnetization reversal, when the amplitude of the buckling mode becomes high enough, one or several vortices nucleate within the particle. However, for an elongated particle the vortex pattern is unstable so that all vortices annihilate during further evolution of the magnetization pattern according to the Landau-Lifshitz-Gilbert equation. As a result, the shape of the hysteresis loop for an elongated elliptical particle is rectangular. It is typified by loop (1) in Fig. 2 for an elliptical particle with aspect ratio $L_{x} / L_{y}=3$.

It is found also that the nucleation field of an elongated elliptical particle saturates in the limit of 
$L_{x} / L_{y} \gg 1$, as shown in Fig. 3 where the nucleation field of an elliptical particle is calculated as a function of particle length at fixed width and thickness. In Fig. 3, the upper bound to the nucleation field corresponds to the value of a reversed external magnetic field under which the quasi-uniform magnetization of the particle is certainly stable. The lower bound gives the value of the external magnetic field under which the quasi-uniform magnetization becomes unstable during the time evolution according to the Landau-Lifshitz-Gilbert equation. The saturation of the nucleation field of the elliptical element is in qualitative agreement with the experimental data [7]. It is a consequence of the fact that the demagnetizing field near the middle part of an elliptical particle decreases as a function of its length. As a result, with increase in the particle length the particle nucleation field approaches that of a very long bar with the same transverse sizes [11].

On the other hand, for the elliptical particle with small enough aspect ratio, the flower state instability occurs in the positive field when the external magnetic field decreases after particle saturation. The shape of the instability mode is close to one of the bending states discussed in Section 2. In addition, for this particle the bending state turns out to be stable in some interval of the external magnetic field. With further decrease of external magnetic field, one or several vortices nucleate within the particle. However, in contrast to the case of the elongated particle, the vortex structure can be stable in some interval of the external magnetic field depending on the particle aspect ratio. As a result, the shape of the hysteresis loop of the particle with small aspect ratio deviates considerably from that of the rectangular one. A typical example for this behavior is presented by loop (2) in Fig. 2. The bending state originates at point A in this loop and remains stable within the interval $A B$, whereas the 1-vortex state exists within the interval CD.

At first glance, the behavior of an elliptical particle resembles that of a rectangular one [3]. However, the important difference is that for a rectangular particle the characteristic field for vortex annihilation is very high, of the order of saturation magnetization, because the vortex can be trapped near the rectangular angle due to the large value of a demagnetizing field in this region [4]. Evidently, this situation does not depend on the particle size or aspect ratio. On the contrary, there is a possibility to control the magnetization reversal process in the elliptical particle to a certain extent. First of all, as we discussed above, the region for stability of the quasiuniform magnetization increases rapidly as a function of the particle aspect ratio. As a result, for the elongated elliptical particle the bending instability occurs in a large negative magnetic field and the interval for the mode stability is practically absent. Next, for the elongated elliptical particle the field for vortex annihilation is rather low, at least in this case it is directed along the

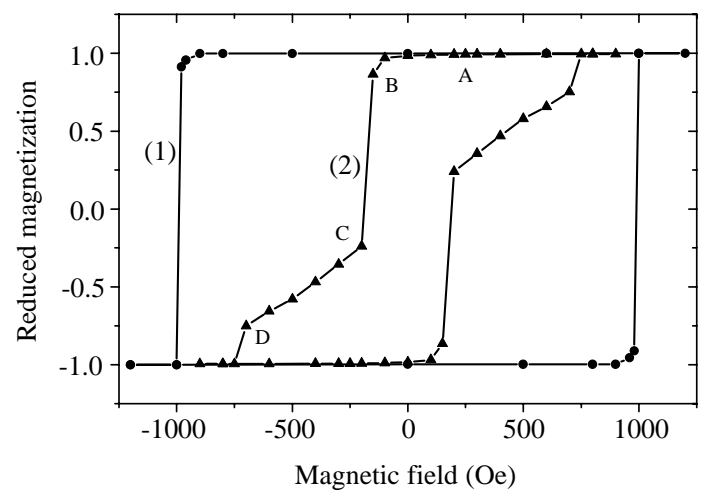

Fig. 2. Hysteresis loops of elliptical particles with thickness $L_{z}=30 \mathrm{~nm}$, length $L_{x}=360 \mathrm{~nm}$ and different aspect ratios: (1) $L_{x} / L_{y}=3$; (2) $L_{x} / L_{y}=1.5$. External magnetic field is parallel to the long particle axis.

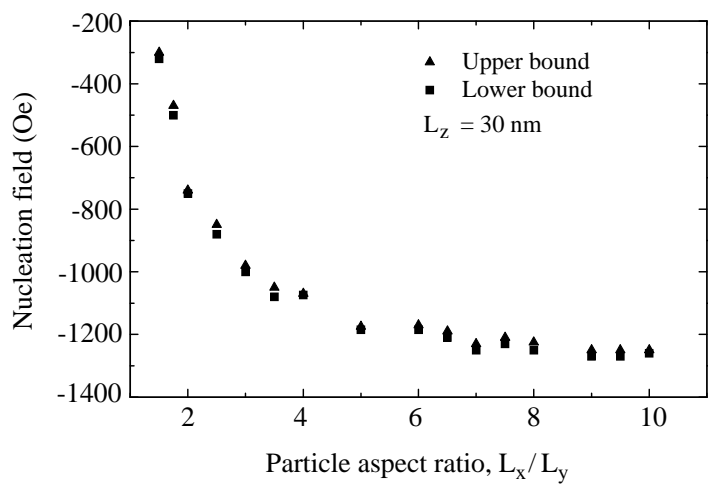

Fig. 3. Nucleation field of an elliptical particle with thickness $L_{z}=30 \mathrm{~nm}$ and width $L_{y}=120 \mathrm{~nm}$ as a function of the particle aspect ratio.

long particle axis [8]. Therefore, choosing properly the particle size and aspect ratio one can shift the onset of the instability to the field larger in absolute value than the characteristic field for vortex annihilation. Thus, in this case the vortices may participate in the magnetization reversal as transient magnetization states only and the hysteresis loop of the particle is rectangular.

\section{Patterned structures}

For an isolated particle the evolution of the magnetization distribution in an external magnetic field can be investigated in detail by means of a numerical simulation. However, some physically plausible approximation is necessary to take into account the effect of mutual magnetostatic interaction on the properties of an array of soft magnetic particles. The idea is to construct a system of point magnetic chargers creating, within the volume of a given particle, a magnetic field close to the 
actual stray magnetic field of a neighboring particle. It can be shown that the stray magnetic field of a cylindrical particle can be approximated by means of a magnetic field of macro-dipole located at the particle center. The macro-dipole consists of two-point magnetic charges of opposite signs, $\pm q_{\mathrm{m}}$, separated from each other by a distance $2 L_{\text {dip }}$. The half-length of the macrodipole is assumed to be constant, $L_{\mathrm{dip}}=\pi R / 4, R$ being the particle radius. Then, the effective magnetic charge of the macro-dipole can be calculated by means of the relation $q_{\mathrm{m}}=\langle m\rangle / 2 L_{\text {dip }}$, where $\langle m\rangle$ is the total magnetic moment of the particle. To justify this approach, the data of exact numerical simulation for two interacting particles were compared with the corresponding results obtained by means of the macrodipole approximation. The macro-dipole approximation was found to be valid for cylindrical particle being in the quasi-uniform or vortex states even for large deviations of the vortex center from the particle center. Using the macro-dipole approximation, the magnetization curves and hysteresis loops of a rectangular array of cylindrical particles with different periods have been studied. Various directions of the in-plane external magnetic field with respect to the array axes were considered. To calculate the magnetic properties of the array the assumption was made that the magnetic states of identical particles of the array are the same at every given value of the external magnetic field. Therefore, it is sufficient to calculate magnetization distribution within the isolated particle subjected to the stray magnetic field of all other particles of a given cluster or array. At every step of the iteration process, one can calculate the total particle magnetic moment and to determine the effective magnetic charge of the macro-dipole using the relation discussed above. In turn, it enables one to calculate in the macro-dipole approximation the total magnetic field of all particles of the array acting on the given particle. If this self-consistent procedure converges, it gives equilibrium array magnetization at every given value of the external magnetic field. Note that Ewald-type summation [12] can be used to take into account slowly decreasing contributions of remote particles to the magnetostatic field acting on the given particle of the array.

An example of the calculations performed is presented in Fig. 4 where the hysteresis loops for a square array of soft cylindrical particles are shown for different orientations, $\varphi_{\mathrm{H}}$, of an in-plane external magnetic field with respect to the $x$-axis of the array. The period of the array, $l=210 \mathrm{~nm}$, is taken to be close to the particle diameter, $D=200 \mathrm{~nm}$, so that the gap between the neighboring particles is small, $d=10 \mathrm{~nm}$. Initially, all the particles of the array are in the lowest energy state, i.e. one-vortex state, the array magnetization being zero in the absence of the external magnetic field. The array magnetization curves for different directions of external
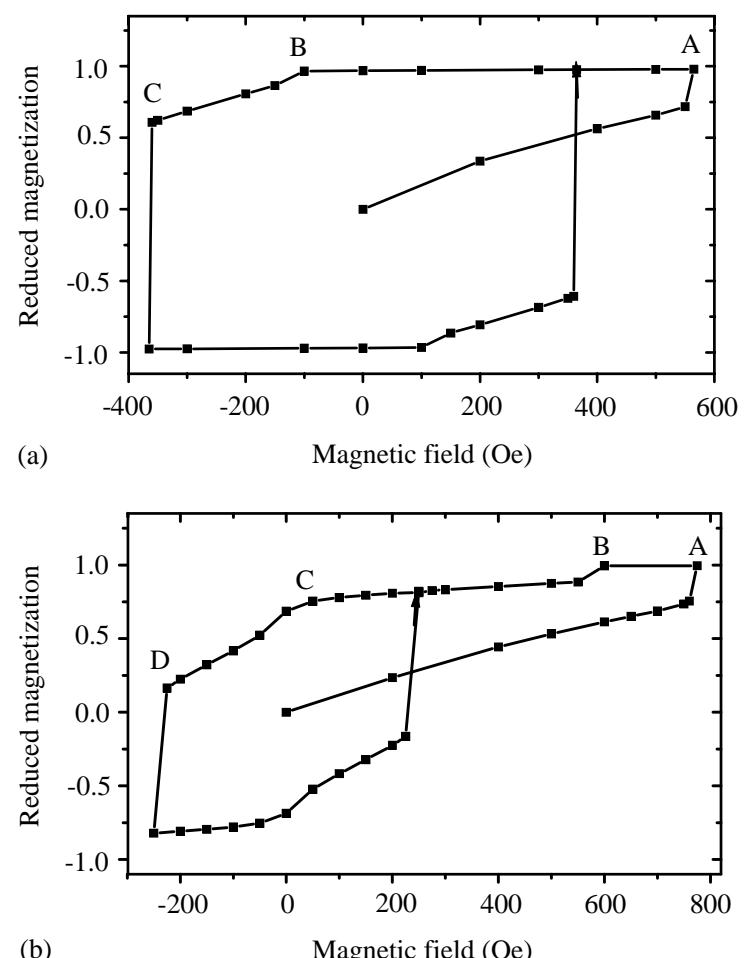

Fig. 4. Magnetization curves and hysteresis loops of square array of Permalloy cylindrical particles with diameter $D=200 \mathrm{~nm}$ and thickness $L_{z}=30 \mathrm{~nm}$ for various directions of external magnetic field with respect to the array axes: (a) $\varphi_{\mathrm{H}}=0$; (b) $\varphi_{\mathrm{H}}=\pi / 4$.

magnetic field are presented in Figs. $4 \mathrm{a}$ and $\mathrm{b}$. It is important to note that for a dense assembly the mutual magnetostatic interaction leads to appreciable decrease of the characteristic magnetic field for array saturation. Actually, the saturation field for isolated Permalloy particle of the same size is given by $H_{0} \approx 1100 \mathrm{Oe}$. Therefore, it is approximately twice higher than that of the square array shown in Fig. 4a. Figs. $4 a$ and b demonstrate also the orientational dependence of the hysteresis loop of the square array. In the case $\varphi_{\mathrm{H}}=0$ the hysteresis loop of the square array is nearly rectangular, the coercive force is given by $H_{\mathrm{c}} \approx 375 \mathrm{Oe}$. When external magnetic field decreases after the array saturation, the nucleation of the BS 2 configuration occurs within the representative particle at point B in Fig. 4a. This configuration remains stable up to point $\mathrm{C}$ in Fig. 4a where two vortices nucleate within the particle. However, the two-vortex state turns out to be only a transient one so that the vortices annihilate and magnetization reversal occurs in one jump. Similar evolution of the BS 2 configuration determines also the magnetic properties of a square array in the case of $\varphi_{\mathrm{H}}=\pi / 4$, with the difference that within the interval CD in Fig. $4 \mathrm{~b}$ the magnetization pattern rotates gradually 
with respect to the magnetic field direction. The coercive force of the square array in the case of $\varphi_{\mathrm{H}}=\pi / 4$ is given by $H_{\mathrm{c}} \approx 250$ Oe.

In conclusion, the calculations performed show considerable dependence of the array saturation field on the array period at a sufficiently small separation between the neighboring particles. It seems interesting to reveal this dependence in the experiment.

\section{Acknowledgements}

The work at TRINITI (Troitsk) was supported partly by means of INTAS grant \#97-31311.

\section{References}

[1] J.M. Daughton, J. Magn. Magn. Mater. 192 (1999) 334.

[2] K.J. Kirk, S. McVitie, J.N. Chapman, C.D.W. Wilkinson, J. Appl. Phys. 89 (2001) 7174.
[3] K.J. Kirk, M.R. Scheinfein, J.N. Chapman, S. McVitie, M.F. Gillies, B.R. Ward, J.G. Tennant, J. Phys. D 34 (2001) 160.

[4] Jing Shi, S.Tehrani, T. Zhu, Y.F. Zheng, J.-G. Zhu, Appl. Phys. Lett. 74 (1999) 2525.

[5] K.J. Kirk, J.N. Chapman, S. McVitie, P.R. Atchison, C.D.W. Wilkinson, Appl. Phys. Lett. 75 (1999) 3683.

[6] K.J. Kirk, J.N. Chapman, C.D.W. Wilkinson, J. Appl. Phys. 85 (1999) 5237.

[7] J.A. Johnson, M. Grimsditch, V. Metlushko, P. Vavassori, B. Ilic, P. Neuzil, R. Kumar, Appl. Phys. Lett. 77 (2000) 4410.

[8] N.A. Usov, Ching-Ray Chang, Zung-Hang Wei, J. Appl. Phys. 89 (2001) 7591.

[9] M.E. Schabes, J. Magn. Magn. Mater. 95 (1991) 249.

[10] N.A. Usov, S.E. Peschany, J. Magn. Magn. Mater. 130 (1994) 275

[11] N.A. Usov, Ching-Ray Chang, Zung-Hang Wei, J. Appl. Phys., submitted for publication.

[12] P. Ewald, Ann. Phys. 64 (1921) 250. 